\title{
ON THE POTENTIAL OF CERTAIN SURFACE DISTRIBUTIONS
}

\author{
BY HILLEL PORITSKY*
}

The surface distributions that are commonly studied in potential theory are those known as single and double layers, or as surface distributions of poles and doublets. A. Wangerin $\dagger$ first investigated potentials of what he called triple layers (dreifach belegte Flächen), that is, potentials of the form

$$
v(P)=\int \mu(\Pi) \frac{\partial^{2}[1 / r(P, \Pi)]}{\partial n^{2}} d S .
$$

Here $r(P, \Pi)$ is the distance between a point $\Pi$ of the surface $S$ and a point $P$ in space, $d S$ is the element of area of $S$ at $\Pi$, $\mu(\pi)$ is a given function along $S$ (the density function), and $\partial / \partial n$ indicates differentiation along the normal toward a specified side of $S$ that might be called its positive side. $\ddagger$

He showed that both $v$ and its first derivatives are discontinuous at $S$. M. Freund $\S$ studied more complex distributions over a spherical surface of the form

$$
v(P)=\int \mu(\Pi) \frac{\partial^{k}[1 / r(P, \Pi)]}{\partial n^{k}} d S .
$$

It is the object of this paper to point out that by means of certain integrations by parts these integrals may be replaced, in case the surface $S$ is closed, by other surface integrals

* National Research Fellow in Mathematics.

$\dagger$ A. Wangerin, Über das Potential dreifach belegter Flächen, Jahresbericht der Vereinigung, vol. 29 (1920), p. 174.

¥ The considerations that lead to the name triple layers are analogous to the ones that suggest the double layer nomenclature: if the derivative is replaced by the limit of a proper quotient, $v$ is seen to be the limit of the potential of three single layers spread over three nearby surfaces.

$\S \mathrm{M}$. Freund, Über das Potential mehrfach belegter Flächen, Dissertation, Universität Halle (1922). 
that represent the potential of certain single and double layers; if, however, the surface $S$ is open, these integrations by parts lead to a replacement of the original distributions by curve distributions over the boundary of $S$ in addition to the above single and double surface layers. Similar conclusions apply to the more general integrals

$$
v(P)=\int \mu(\Pi) \frac{\partial^{k}[1 / r(P, \Pi)]}{\partial h_{1} \partial h_{2} \cdots \partial h_{k}} d S,
$$

where $\partial / \partial h_{i}, i=1,2, \cdots, k$, indicate differentiations in $k$ specified directions at each point $\Pi$ of $S$, so that for a fixed $\Pi$ the integrand is a spherical harmonic of degree $-(k+1)$ in the coordinates of $P$ when the origin is at $\Pi$. From this it would appear that except for features presented at the boundary, if any, of $S$, the surface distributions of the above types introduce no new element into potential theory.

This reduction to simpler surface distributions may be carried out as follows. Introduce a triply orthogonal family of surfaces of which $S$ is one by taking for one family the surfaces parallel to $S$ and for the other two the developable surfaces consisting of the normals to $S$ through its lines of curvature. Let $u, v$ be the parameters along the latter, while $n$ is the (directed) distance from $S ; u, v, n$ may be used as parameters for the above surfaces or as curvilinear coordinates for points near $S$. Let the element of distance be given by

$$
d s^{2}=g_{1}^{2} d u^{2}+g_{2}^{2} d v^{2}+d n^{2} ;
$$

$g_{1}, g_{2}$ are completely determined by their values for $n=0$. Indeed,

$$
g_{i}(u, v, n)=g_{i}(u, v, 0)\left(1+n / R_{i}(u, v)\right), \quad(i=1,2,)
$$

where $R_{1}, R_{2}$ are the radii of curvature along $v=$ const., $u=$ const. respectively, measured positively if the corresponding centers of curvature are on the side of negative $n$. Using 
the well known expression for the Laplacian in orthogonal coordinates, we get

$$
\begin{aligned}
\nabla^{2}=\left\{\frac { 1 } { g _ { 1 } g _ { 2 } } \left[\frac{\partial}{\partial u}\left(\frac{g_{2}}{g_{1}} \frac{\partial}{\partial u}\right)+\right.\right. & \left.\left.\frac{\partial}{\partial v}\left(\frac{g_{1}}{g_{2}} \frac{\partial}{\partial v}\right)\right]\right\} \\
+ & \left\{\frac{1}{g_{1} g_{2}} \frac{\partial}{\partial n}\left(g_{1} g_{2} \frac{\partial}{\partial n}\right)\right\}
\end{aligned}
$$

If using (5) we form the equation $\nabla^{2}(1 / r)=0$, where $r$ is the distance from a fixed point $P$ not on $S$, we obtain an equation solvable for $\partial^{2}(1 / r) / \partial n^{2}$ in terms of first and second order derivatives of $1 / r$ among which not more than one $n$-differentiation occurs. In a similar way, by the use of this equation and of the equations that are obtained from it by differentiating it with respect to $n$ once, twice, $\cdots,(k-2)$ times, one could express $\partial^{k}(1 / r) / \partial n^{k}$ in terms of $u$-, $v$-derivatives of $1 / r$ and of $\partial(1 / r) / \partial n$. Likewise, the spherical harmonic $\partial^{k}(1 / r) / \partial h_{1} \cdots \partial h_{k}$ in the integrand of (3) may be expressed in terms of such differentiations as follows. Introduce rectangular Cartesian coordinates $x, y, z$ and replace $\partial / \partial h_{i}$ by $\cos \left(x h_{i}\right) \partial / \partial x+\cos \left(y h_{i}\right) \partial / \partial y+\cos \left(z h_{i}\right) \partial / \partial z$ (the parentheses denote angles between the direction $h_{i}$ and the positive $x, y, z$ axes), and transform to $u$-, $v$-, $n$-derivatives by means of elementary formulas for change of derivatives under a transformation of variables. The $n$-derivatives of second or higher order may now be eliminated from the result by the use of the equations obtained from $\nabla^{2}(1 / r)=0$ (expressed in the form (5)) by differentiating it the requisite number of times with respect to $u, v, n$.

Having thus expressed the integrands in (1), (2), (3), we may then integrate the resulting integrals by parts enough times to make all the $u$-, $v$-derivatives of $1 / r, \partial(1 / r) / \partial n$ disappear from the remaining surface integrals. The resulting surface integrals can be collected into the form

$$
\int a(1 / r) d S+\int b[\partial(1 / r) / \partial n] d S
$$


where $a, b$ are derived from $\mu$ by operating on it with certain linear differential operators that depend upon the shape of $S$ near $\Pi$ but are independent of $P$. These surface integrals, therefore, represent potentials of a single and of a double surface layer. If the surface $S$ is open, the integrations by parts will also introduce certain integrals over the bounding curves that represent potentials of poles, doublets, and more complex singularities (in cases (2), (3)) distributed over these boundaries. Such curve distributions cannot, in general, be reduced to simpler curve distributions.*

For the case of the triple layers of Wangerin the above computations may be simplified by the use of the well known Green-Beltrami integration theorems. Returning to (5) we recognize in the first brace the second differential operator of Beltrami (see W. Blaschke, Vorlesungen über Differentialgeometrie, vol. I, p. 116 (121)); we shall denote it by $B^{2}$. Expanding the second brace and using (4) we get for the Laplacian

$$
\nabla^{2}=B^{2}+2 H \partial / \partial n+\partial^{2} / \partial n^{2},
$$

where $H=(1 / 2)\left(1 / R_{1}+1 / R_{2}\right)$ is the mean curvature. The elimination of $\partial^{2}(1 / r) / \partial n^{2}$ from the integrand of (1) outlined above results in the equation

$$
v(P)=-\int B^{2}(1 / r) \mu d S-\int 2 H \mu[\partial \mu(1 / \boldsymbol{r}) / \partial n] d S .
$$

The first integral may now be transformed by the integral relation of Beltrami

$$
\int\left(f B^{2} g-g B^{2} f\right) d S=\int\left(f \frac{\partial g}{\partial \xi}-g \frac{\partial f}{\partial \xi}\right) d C
$$

(see Blaschke, loc. cit. p. 117 (129); we denote the element

* It may be pointed out in this connection that the potential of a volume distribution of singularities of the type (3) can always, for points outside the region of integration, be reduced to a potential of a volume distribution of poles only, in addition to surface distributions of the form (3). A familiar instance of this is furnished by Poisson's theory of magnetism. 
of arc length by $d C$ and the derivative along the outer normal to $C$ by $\partial / \partial \xi)$. We get

$$
\begin{aligned}
v(P)= & -\int B^{2} \mu(1 / r) d S-\int 2 H \mu[\partial(1 / r) / \partial n] d S \\
& -\int \mu[\partial(1 / r) / \partial \xi] d C+\int(1 / r)(\partial \mu / \partial \xi) d C .
\end{aligned}
$$

Hence, the density of the single layer is $-B^{2} \mu$ and that of the double layer $-2 H \mu$. The results of Wangerin for the discontinuities in $v$ and its derivatives at the surface $S$ readily follow from the known properties of single and double layers.*

Without going through the explicit computation of the densities of the equivalent single and double surface layers in cases (2), (3), we can tell from the mere existence of these layers that for approach of an inner point of $S$ along the normal, these potentials approach limits which depend, in general, upon the side of approach. This even sheds some light on the behavior near $S$ of higher order derivatives (with respect to Cartesian variables) of the familiar single and double layer potentials, since such derivatives may be written in the form (3); $†$ such a form may also be given to derivatives of any order of the potentials (1), (2), (3).

We conclude with a simple example that will serve to illustrate the behavior of these potentials of distributions over open surfaces near the edge of the surface. Consider a distribution of the form (3) over an infinite plane strip, say $y=0,0<x<a$, with the $k$ directions $h_{1}, \cdots, h_{k}$ constant and $\mu$ depending only on the distance from the edge. Expressing the directional derivatives in terms of derivatives

\footnotetext{
* But the converse is in a sense also true. Thus, if $S$ is closed, $v$ is uniquely determined if it is harmonic except on $S$, if it and its derivative along the normal approach limits for approach of $S$ from opposite sides and if a certain behavior is ascertained at infinity.

$\dagger$ The explicit values of the discontinuities for second-order derivatives of potentials of single layers have been obtained by Poincaré in his Theorie $d u$ Potentiel Newtonien, \$105, etc.
} 
with respect to $x, y, z$ and integrating with respect to $z$ first, we get for $v$ a sum of terms of the form*

$$
\begin{gathered}
\int_{0}^{a} \mu(x) \frac{\partial^{k} \log r}{\partial x^{l} \partial y^{k-l}} d x \\
=\int_{0}^{a} \mu(x) R\left(\frac{\partial^{k} \log (z-h)}{\partial x^{l} \partial y^{k-l}}\right) d x,
\end{gathered}
$$

where $z$ is the complex quantity $x+i y, h$ is the value of $z$ at $P$ and $R(\alpha)$ denotes the real part of $\alpha$. But by the CauchyRiemann equations for an analytic function $F$ of a complex variable

$$
\frac{\partial F}{\partial x}=\frac{\partial F}{i \partial y}=F^{\prime}(z) .
$$

Hence the integral becomes

$$
R \int_{0}^{a} i^{k-l} \mu(x) \frac{\partial^{k} \log (z-h)}{\partial x^{k}} d x,
$$

and, integrating by parts $k$ times, we obtain an integral that represents a bounded function of $h$ for $|h|<$ const. and integrated terms that yield singularities at $h=0, h=a$. The terms resulting from the lower limit of integration are given by $R\left(c_{0} \log h+c_{1} / h+c_{2} / h^{2}+\cdots+c_{k-1} / h^{k-1}\right)$ where the $c_{i}$ are constants; similar terms result from the upper limit.

\section{HARVARD UNIVERSITY}

* This furnishes an example of a two-dimensional potential of singularities analogous to those of (3) for space. The general treatment of such potentials could of course be given along similar lines or could be deduced from the above by putting $1 / R_{2}=0$. 